\title{
Adenosquamous Carcinoma of the Pancreas That Had Penetrated Into the Stomach and Transverse Colon: A Case Report
}

\author{
Nobuhiro Takeuchi ${ }^{\mathrm{a}, \mathrm{c}}$, Kazumasa Emori ${ }^{\mathrm{a}}$, Makoto Yoshitani ${ }^{\mathrm{a}}$, \\ Junichi Soneda ${ }^{a}$, Kaori Mohrib
}

\begin{abstract}
We report the case of pancreatic adenosquamous carcinoma (PASC) that had penetrated into the stomach and transverse colon. A 55-yearold male presented to our hospital with general fatigue, melena, and left flank pain. He had no relevant medical history. A laboratory analysis upon arrival revealed marked anemia with a red blood cell count of $13.3 \times 10^{4} / \mu \mathrm{L}$ and a hemoglobin level of $3.6 \mathrm{~g} / \mathrm{dL}$. There was tenderness over the upper abdomen and a palpable mass on his left upper abdomen. He was admitted for further examination of his severe anemia and abdominal symptoms. Contrast-enhanced computed tomography revealed multiple liver masses and a $10 \mathrm{~cm}$ sized tumor in the pancreas tail involving the gastric wall and transverse colon. Upper gastroendoscopy revealed a $4 \mathrm{~cm}$ sized elevated tumor with a central depression in the greater curvature of the middle gastric body. Colonoscopy revealed an ulcerative tumor extending from the splenic flexure to the mid transverse colon. Endoscopic biopsied specimens revealed adenosquamous cell carcinoma. From these findings, the diagnosis of PASC in the pancreatic tail invading into the stomach and transverse colon at an advanced stage IV was established. His anemia was treated by the administration of red blood cell concentration (RCC). Although chemotherapy was planned after his malnutrition improved, his circulatory condition worsened on day 20. Despite 10 units of RCC, his anemia did not improve and he died from circulatory insufficiency on day 23. An autopsy revealed PASC in the pancreas tail measuring $14 \mathrm{~cm}$ that had penetrated into the gastric wall and transverse colon. A massive amount of blood was discovered in the stomach. His death was caused by massive bleeding from the invaded lesions of the stomach. Adenosquamous carcinoma, consisting of adenocarcinoma and squamous cell carcinoma, is characterized by its aggressive proliferation and poor prognosis. To date, consensuses in the management of PASC have not been established. Further accumulation of knowledge about this carcinoma is required.
\end{abstract}

Manuscript accepted for publication December 15, 2016

aDepartment of Internal Medicine, Kobe Tokushukai Hospital, 1-3-10 Kamitakamaru, Tarumi-ku, Kobe-shi, Hyogo 655-0017, Japan

bepartment of Laboratory Medicine, Kobe Tokushukai Hospital, 1-3-10 Kamitakamaru, Tarumi-ku, Kobe-shi, Hyogo 655-0017, Japan

${ }^{\mathrm{c} C}$ Corresponding Author: Nobuhiro Takeuchi, Department of Internal Medicine, Kobe Tokushukai Hospital, 1-3-10 Kamitakamaru, Tarumi-Ku, Kobeshi, Hyogo 655-0017, Japan. Email: nobuhiro.takeuchi@tokushukai.jp

doi: https://doi.org/10.14740/jmc2723w
Keywords: Adenosquamous carcinoma; Pancreas; Perforation

\section{Introduction}

Adenosquamous carcinoma is characterized by the histological presence of both adenocarcinoma and squamous cell carcinoma (SCC) within the same tumor. Compared with adenocarcinoma, adenosquamous carcinoma has a lower incidence and poorer prognosis. A histological analysis reported that pancreatic adenosquamous carcinoma (PASC) comprises only 0.09$0.4 \%$ of all cancers that occurs in the pancreas $[1,2]$. PASC is diagnosed based on the criteria that more than $30 \%$ of squamous components are included in the pancreas tumor tissues [3]. The biological nature of PASC is its aggressive proliferation and invasiveness into other tissues, including the lymph nodes, vessels, and nerve plexuses. Here we report the case of a patient with PASC that had penetrated into the stomach and transverse colon.

\section{Case Report}

A 55-year-old male with no relevant medical history presented with complaints of left flank pain, general fatigue, a sense of listlessness, and melena. His mother and aunt had died of colorectal cancer, and his father had died of lung cancer. He had been experiencing a dull pain in the left flank for 2 months prior to his presentation to the hospital. Upon presentation, severe anemia was evident based on his palpebral conjunctiva. There was tenderness over the upper abdomen and a palpable mass on his left upper abdomen.

Blood chemistry revealed remarkably elevated inflammatory reactions (white blood cell count of $25,250 / \mu \mathrm{L}$, of which $93 \%$ were neutrophils, and a C-reactive protein level of 8.56 $\mathrm{mg} / \mathrm{dL}$ ), severe anemia (red blood cell counts of $13.3 \times 10^{4} / \mu \mathrm{L}$, and a hemoglobin level of $3.6 \mathrm{~g} / \mathrm{dL}$ ), severe hypoproteinemia $(5.2 \mathrm{~g} / \mathrm{dL})$, severe albuminemia $(1.7 \mathrm{~g} / \mathrm{dL})$, mildly elevated gamma-glutamyl transpeptidase $(78 \mathrm{IU} / \mathrm{L})$, mildly elevated lactate dehydrogenase (245 IU/L), mild hyponatremia (131 $\mathrm{mEq} / \mathrm{L}$ ), and a mild coagulation disorder $(73 \%$ prothrombin and D-dimer level of $1.9 \mu \mathrm{g} / \mathrm{mL}$ ). His carcinoembryonic antigen level was moderately elevated at $37.9 \mathrm{ng} / \mathrm{mL}$, and the can- 

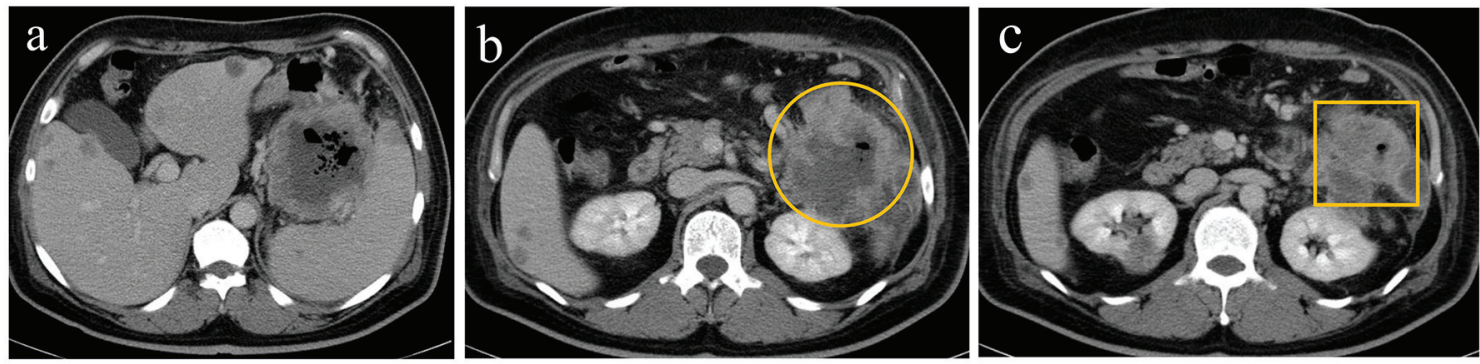

Figure 1. Contrast-enhanced computed tomography revealed a marked thickening of the gastric walls along with multiple liver metastases (a), a cystic mass surrounded by contrast-enhanced margins (circle) (b), and thickening of the walls in the transverse colon (square) (c)

cer antigen 19-9 (CA 19-9) was remarkably elevated at 1,977 $\mathrm{U} / \mathrm{mL}$. The SCC antigen and soluble interleukin-2 receptor, which are two tumor markers, were moderately elevated at $29.8 \mathrm{ng} / \mathrm{mL}$ and $1,165 \mathrm{U} / \mathrm{mL}$, respectively.

Contrast-enhanced computed tomography (CT) revealed multiple liver metastases and thickened gastric walls (Fig. 1a). A cystic tumor was apparent in the pancreatic tail, with invasion into the transverse colon (Fig. 1b). Ultrasonography revealed remarkably thickened muscle layers of the gastric body (Fig. 2a) and the cystic tumor in the pancreatic tail (Fig. 2b). Upper gastrointestinal endoscopy revealed a $4.0 \mathrm{~cm}$ sized protruding mass with an ulcer formation in the greater curvature of the middle gastric body (Fig. 3a). Endoscopic biopsies revealed two components of SCC and adenocarcinoma (Fig. $3 b)$. Colonoscopy revealed massive ulcers extending from the splenic flexure to the middle transverse colon (Fig. 3c). Endoscopic biopsies also revealed two components of SCC and adenocarcinoma (Fig. 3d). Based on these findings, this patient was diagnosed with adenosquamous carcinoma of the pancreas that had invaded into the stomach and transverse colon. Although chemotherapy and palliative surgery were considered, the patient was unable to tolerate the treatment until his general condition and malnutrition improved. Administration of a red blood cell concentration (RCC) and intravenous nutrition was provided. On day 20, the patient's blood pressure suddenly deteriorated and his anemia worsened. Although 10 units of RCC were administered, he died of circulatory insufficiency.

An autopsy was performed under the consent of his family. The stomach was perforated and contained $100 \mathrm{~mL}$ of blood (Fig. 4a). A cystic mass measuring $14 \mathrm{~cm}$ was discovered in the pancreatic tail that it penetrated into the gastric and transverse colon walls (Fig. 4b). Histological findings of the pancreatic tumors (Fig. 4c) and invaded gastric lesions (Fig. 4d) confirmed adenosquamous carcinoma with atypical cells accompanied by inflammatory cells and fibrosis proliferation, forming an alveolar and sheet-like structure along with keratinization, an intercellular bridge, and glandular formation. Based on these findings, it was concluded that his death was caused by bleeding from the stomach that was invaded by the PASC.

\section{Discussion}

Adenosquamous carcinoma characterized by the presence of adenocarcinoma and SCC in the same tumor is known to have a poor prognosis and an aggressive proliferation. According to the current guidelines, PASC is defined by the presence of both adenomatous and squamous components, in which the latter comprises over $30 \%$ of the tumors [3]. Adenosquamous carcinoma occurs in the esophagus, stomach, colon, pancreas, biliary tract, liver, salivary glands, and female reproductive system [4]. Besides the aggressiveness and invasiveness of adenosquamous carcinoma, in general, there is little difference between PASC and pancreatic ductal adenocarcinoma in its clinical manifestation, including age, sex, location, size, and primary symptoms.

The mucous membranes in the pancreatic duct have no
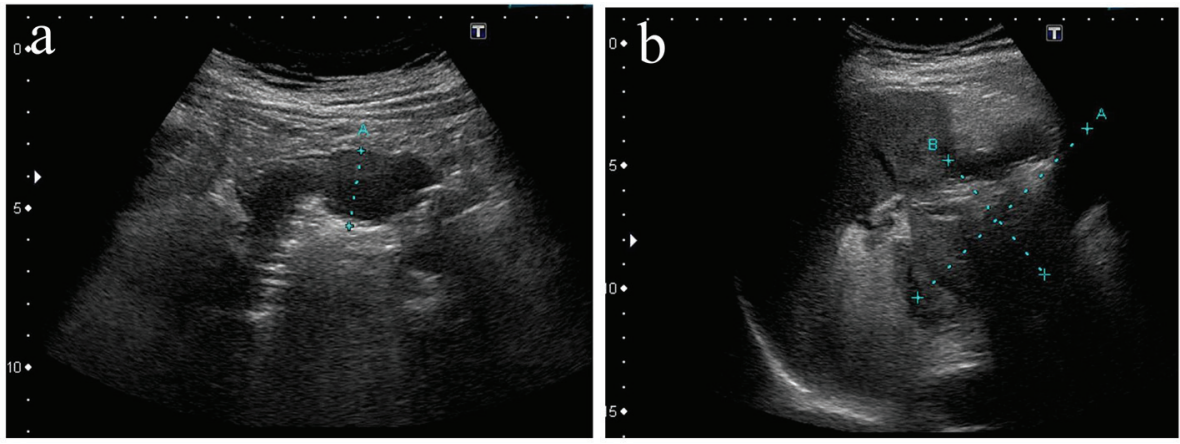

Figure 2. Ultrasonography revealed thickening of gastric muscle layers characterized by low echoic lesions (a) and a cystic mass in the pancreas tail (b). 

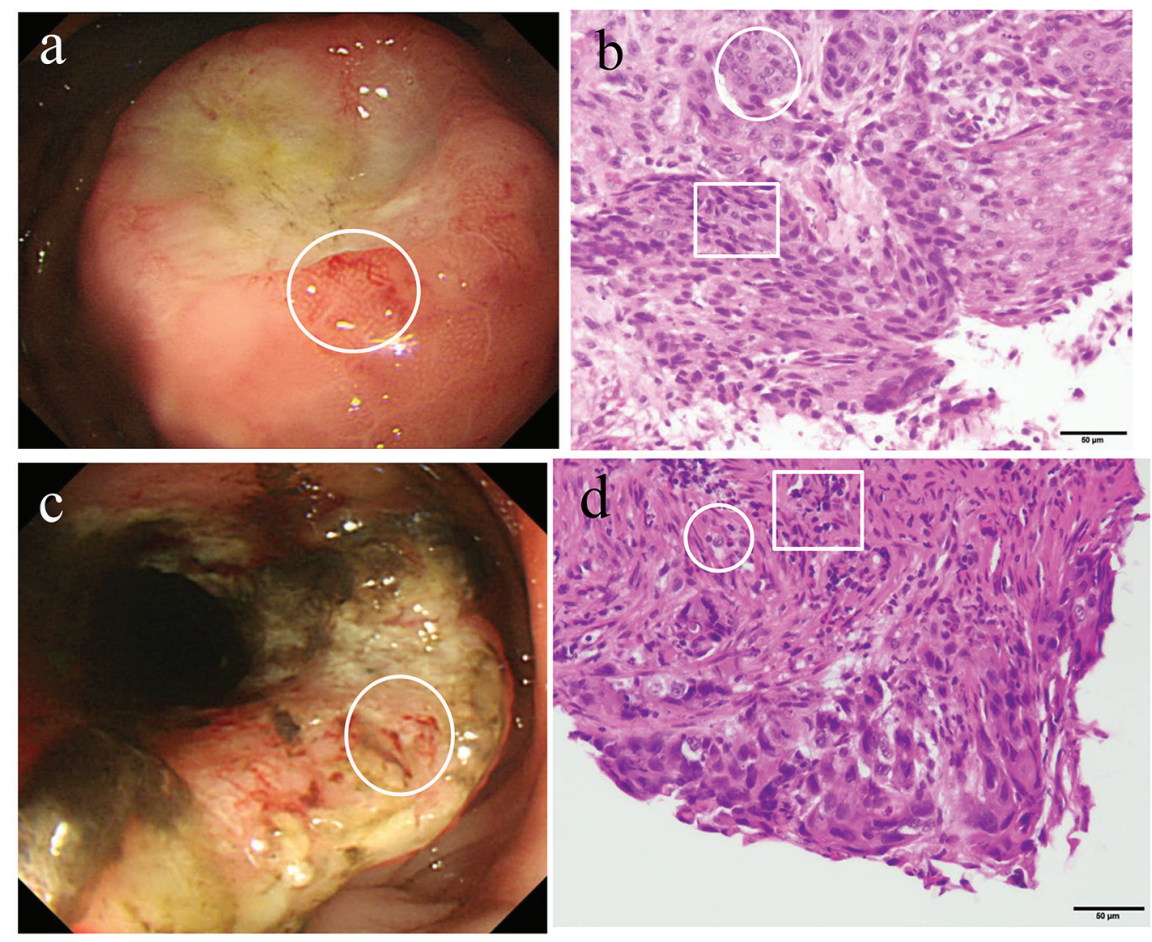

Figure 3. Upper gastric endoscopy revealed a raised mass with central depressive lesions in the middle body of the greater curvature (a). A biopsied specimen of the gastric tumor (circle) revealed the presence of both adenocarcinoma (circle) and squamous cell carcinoma (square) (b). A colonoscopy revealed a half-circumferential lesion in the splenic flexure of the transverse colon (c). A biopsied specimen (circle) revealed the presence of both adenocarcinoma (circle) and squamous cell carcinoma (square) (d).
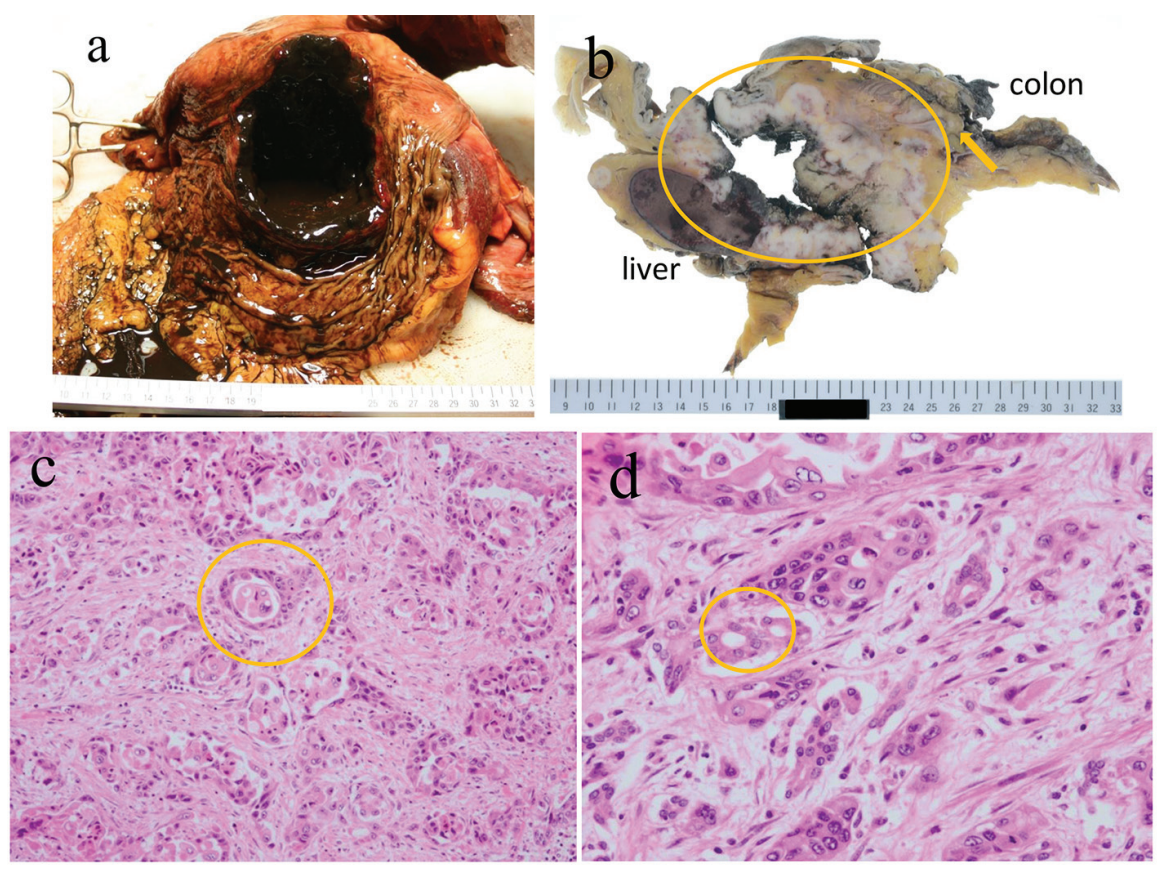

Figure 4. An autopsy revealed that the stomach was perforated and contained $100 \mathrm{~mL}$ of blood (a). The perforation site between the pancreatic tumor in the tail and the transverse colon (arrow indicates perforation site; circle indicates the pancreatic tumor) (b). Histological findings of pancreatic tumors (c) and invaded gastric lesions (d) revealed atypical cells accompanied by inflammatory cells and prolific fibrosis, making an alveolar and sheet-like structure, along with keratinization, an intercellular bridge, and a glandular formation. 
squamous epithelium, and SCC is hypothesized to originate from glandular tissues. There are four proposed mechanisms for the development of adenosquamous carcinoma: 1) ectopic squamous epithelium, 2) squamous metaplasia of the pancreatic ductal epithelium, 3) squamous metaplasia of the glandular system, and 4) histological transformation of adenocarcinoma. To date, evidence from many studies suggests that a histological transformation of adenocarcinoma to SCC 4) might be the underlying mechanism [5-7]. However, considering that there are some cases of early-stage squamous carcinoma without an adenomatous component, 1)-3) mechanisms are also possible.

The squamous component of this type of tumor was reported to proliferate two times faster than its adenomatous component (doubling time of squamous component is 81.8 days, whereas that of adenomatous component is 166.3 days) [8]. The rapid growth of squamous components in PASC is presumably related with bleeding or cystic and necrotic transformations of the tumor. The compression of adenomatous components by squamous cell proliferation leads to the loss of a distinct border between the two components. Furthermore, as the tumor progresses, squamous cell proliferation continues to replace adenocarcinoma. Tumors with a higher percentage of squamous components tend to progress more rapidly and have a poorer prognosis. It is notable that expansive growth is a characteristic of PASC.

In general, adenosquamous carcinoma has a poor prognosis compared with cancers with other histological origins in the pancreas because of its tendency to aggressively invade the lymph nodes, vessels, and nerve plexuses [9]. Thus, the prognosis of PASC is generally considered to be poor; the average survival period of patients undergoing surgical resection is approximately 6 months and that of those who underwent palliative treatment is 1.5 months.

The characteristics of an imaging diagnosis are rich vessel proliferation and cystic transformation accompanied by internal necrosis. Contrast-enhanced CT of PASC revealed contrast-enhanced margins accompanied by non-contrastenhanced lesions inside, suggesting hemorrhage or necrosis changes [10]. This finding is effective for differentiating PASC from pancreatic ductal carcinoma. In our patient, contrastenhanced CT revealed a cystic tumor with contrast-enhanced margins, which was indicative of PASC.

The laboratory characteristic of PASC is increased level of serum CA19-9. It may be helpful to assess serum CA 19-9 levels in pancreatic tumors that have different clinical or imaging characteristics from pancreatic ductal adenocarcinoma.

The treatment of PASC is a curative resection in patients without distant metastases; however, few PASC cases are discovered at an early stage because of its aggressive proliferation, making them unsuitable candidates for a surgical resection. A surgical resection of a localized PASC is controversial because of its worse prognosis after the resection than that without surgery; however, it is certain that some patients with PASC can benefit from a longer survival time after the surgical treatment [11].

There are a few cases reported in Japan where the patient survived for more than 5 years after the surgical resection of PASC. The mainstay of PASC is chemotherapy. However, there are currently no compromised protocols for treating
PASC. PASC regimens are proposed in accordance with those of pancreatic adenocarcinoma, including a monotherapy of $\mathrm{S}-1$, or a combination therapy of S-1 plus gemcitabine, or an S-1 plus platinum- and/or a paclitaxel-based chemotherapy [12-15]. Squamous carcinoma is considered to be susceptible to radiation therapy, and postoperative radiation therapy has been found to yield some favorable results [16].

This present patient was deemed unsuitable for chemotherapy or radiotherapy because of his poor performance status and malnutrition. If he had survived until his general condition had improved enough to be able to tolerate chemotherapy, there was a little hope for longer survival.

\section{Conclusion}

We herein reported the case of a patient with PASC that had penetrated into the stomach and transverse colon. Although PASC is characterized as having a poor prognosis because of its rapid and aggressive growth, efficient and compromised treatment strategies have yet to be established. In advanced PASC stages that are unsuitable for a surgical resection, chemotherapy, radiotherapy, or a combination of both therapies are attempted; further accumulation of experiences in treating PASC is required.

\section{References}

1. Fitzgerald TL, Hickner ZJ, Schmitz M, Kort EJ. Changing incidence of pancreatic neoplasms: a 16-year review of statewide tumor registry. Pancreas. 2008;37(2):134138.

2. Simone CG, Zuluaga Toro T, Chan E, Feely MM, Trevino JG, George TJ, Jr. Characteristics and outcomes of adenosquamous carcinoma of the pancreas. Gastrointest Cancer Res. 2013;6(3):75-79.

3. Kardon DE, Thompson LD, Przygodzki RM, Heffess CS. Adenosquamous carcinoma of the pancreas: a clinicopathologic series of 25 cases. Mod Pathol. 2001;14(5):443-451.

4. Madura JA, Jarman BT, Doherty MG, Yum MN, Howard TJ. Adenosquamous carcinoma of the pancreas. Arch Surg. 1999;134(6):599-603.

5. Cao SH. Extrahepatic bile duct cancer. Report of 106 cases. Chin Med J (Engl). 1992;105(8):622-629.

6. Iemura A, Yano H, Mizoguchi A, Kojiro M. A cholangiocellular carcinoma nude mouse strain showing histologic alteration from adenocarcinoma to squamous cell carcinoma. Cancer. 1992;70(2):415-422.

7. Momosaki S, Yano H, Kojiro M. Establishment of a human hepatic adenosquamous carcinoma cell line (KMC-2) and its response to cytokines. Pathol Int. 1995;45(2):137146.

8. Charbit A, Malaise EP, Tubiana M. Relation between the pathological nature and the growth rate of human tumors. Eur J Cancer. 1971;7(4):307-315.

9. Hong SM, Kim MJ, Jang KT, Yoon GS, Cho H, Frierson $\mathrm{HF}$, Yu E. Adenosquamous carcinoma of extrahepatic bile 
duct: clinicopathologic study of 12 cases. Int J Clin Exp Pathol. 2008;1(2):147-156.

10. Toshima F, Inoue D, Yoshida K, Yoneda N, Minami T, Kobayashi S, Ikdeda H, et al. Adenosquamous carcinoma of pancreas: CT and MR imaging features in eight patients, with pathologic correlations and comparison with adenocarcinoma of pancreas. Abdom Radiol (NY). 2016;41(3):508-520.

11. Boyd CA, Benarroch-Gampel J, Sheffield KM, Cooksley CD, Riall TS. 415 patients with adenosquamous carcinoma of the pancreas: a population-based analysis of prognosis and survival. J Surg Res. 2012;174(1):12-19.

12. Saso K, Murata M, Asai K, Yamada M, Yagi T, Katsuyama S, Sawami H, et al. [A Case of Pancreatic Cancer with Multiple Liver Metastases That Developed Postoperatively and Showed a Complete Response with S-1 Monotherapy]. Gan To Kagaku Ryoho. 2015;42(12):16201622.

13. Sumiyoshi H, Matsushita A, Nakamura Y, Yamahatsu K,
Katsuno A, Uchida E. [Radical resection of a locally advanced pancreatic tail adenosquamous carcinoma treated with S-1 and gemcitabine as neoadjuvant chemotherapy - a case report]. Gan To Kagaku Ryoho. 2014;41(5):669672 .

14. Kataoka K, Matsubara H, Urano F, Okamura S, Maeda M. Nab-paclitaxel+gemcitabine therapy for adenosquamous carcinoma of the pancreas: an autopsy case. Nihon Shokakibyo Gakkai Zasshi. 2016;113(10):1777-1784.

15. Ikeda E, Shigematsu T, Hidaka K, Nishikawa A, Katayama M, Okajima T, Kawahara T, et al. [A case of adenosquamous gastric carcinoma successfully treated with TS-1, low-dose CDDP and docetaxel as neoadjuvant chemotherapy]. Gan To Kagaku Ryoho. 2007;34(3):423426.

16. Shibagaki K, Fujita K, Nakayama S, Takenaka M, Fukuba N, Matsui S, Ozaka M, et al. Complete response of a pancreatic adenosquamous carcinoma to chemoradiotherapy. Int J Clin Oncol. 2008;13(1):74-77. 